\section{Jules Bordet, \\ un homme \\ de conviction}

\section{Centenaire}

de l'attribution

de son prix Nobel

\section{Jean-Marc Cavaillon ${ }^{1,2}$, Philippe Sansonetti ${ }^{1,3}$, Michel Goldman ${ }^{4}$}

Paradoxalement, il va y déchiffrer certains des mécanismes clés de l'immunité humorale, initialement découverte par l'école allemande. II y décrit notamment les mécanismes qui aboutissent à la bactériolyse et l'hémolyse par l'action d'immunsérums. Même s'il favorisa le terme d'alexine, créé par Hans Buchner, c'est bien le système du complément (terme inventé par Paul Ehrlich) dont il est un des pères fondateurs. C'est pour ces travaux qu'il se verra attribué en octobre 1920 le prix Nobel de physiologie ou médecine millésimé 1919. Il identifia aussi le bacille de la coqueluche, qui porte son nom Bordetella pertussis. <

\section{Les frères Bordet}

Charles (né en 1868) et Jules (né en 1870) étaient les fils de Charles Henri Bordet (1834-1905), professeur d'école, et de Céleste Vandenabeele (1831-1919). Charles avait 18 ans et Jules 16 ans quand ils commencèrent leurs études de médecine à I'Université libre de Bruxelles (ULB) [1]. Pendant leurs études, tous deux vont mener des activités de recherches dans un laboratoire. Charles travaille avec un autre étudiant, Jean Massart (18651925), dans le laboratoire de Paul Héger (1846-1925), médecin et physiologiste qui était directeur du Solvay Research Institute. En utilisant une approche ingénieuse et brillante, ils ont fait des découvertes majeures sur le chimiotactisme [2,3]. En 1892, Jules Bordet publie sa première contribution scientifique issue du laboratoire de Léo Errera (1858-1905), botaniste belge de renom travaillant à l'Université libre de Bruxelles et membre correspondant de l'Académie royale des sciences de Belgique. Son premier travail porte sur l'adaptation d'une bactérie, qui porte le nom du scientifique qui deviendra

Vignette (Photo @ Institut Pasteur-photothèque).

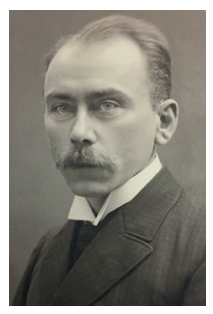

\author{
${ }^{1}$ Institut Pasteur, 28 rue du Docteur Roux, 75015 Paris, \\ France. \\ ${ }^{2}$ Agence national pour la recherche, Paris, France. \\ ${ }^{3}$ Collège de France, Paris, France. \\ ${ }^{4}$ Institute for Interdisciplinary Innovation in healthcare \\ (I3h), Université libre de Bruxelles, Belgique. \\ jean-marc.cavaillon@pasteur.fr
}

son prochain mentor, Vibrio metschnikovii, et découverte par Nicolaï Gamaleïa (1859-1949), venu se former à l'institut Pasteur, et dont les liens avec cet institut demeurent complexes ${ }^{1}$ [4]. Jules Bordet tenta d'expliquer pourquoi la virulence de la bactérie augmente lorsqu'elle est transférée de cobayes immunisés à des cobayes immunisés, une observation qui avait été faite précédemment par Elie Metchnikoff lui-même. Une des hypothèses était que la bactérie pourrait produire un niveau réduit de chimioattractant. Pour répondre à cette question, Jules utilisa le modèle expérimental qui avait été si bien utilisé par son frère et parvint à la conclusion que l'augmentation de la virulence de la bactérie était consécutive à une toxicité moindre et un chimiotactisme réduit [5]. Après avoir obtenu son diplôme de médecine en 1892, Jules Bordet se vit offrir par le gouvernement belge une bourse pour aller travailler à l'Institut Pasteur à Paris. Alors que Charles se lance dans la pratique clinique et arrête toute recherche, cette opportunité offerte à Jules sera décisive pour sa carrière, ses futures découvertes, et pour sa gloire future. Jules Bordet rejoint l'Institut Pasteur en avril 1894. II y suit le «Grand Cours de Microbie » organisé par Émile Roux. Très vite, Jules Bordet devient l'assistant d'Elie Metchnikoff et initie ses propres expériences. Dès 1894, dans son rapport du Congrès international qui s'est tenu à Budapest, Metchnikoff fait référence à des expériences menées par Jules Bordet dans son laboratoire [6]. Bordet a ainsi rejoint le laboratoire du père des phagocytes et de l'immunité cellulaire, alors en conflit avec l'école allemande qui défendait activement l'immunité humorale et qui abhorrait la théorie cellulaire de l'immunité [7]. Étonnamment, Metchnikoff invita Bordet à travailler sur l'immunité humo-

${ }^{1}$ Devant prendre la responsabilité d'une des unités pressenties à la création de l'institut Pasteur, sa venue ne sera finalement jamais validée [4]. 
A

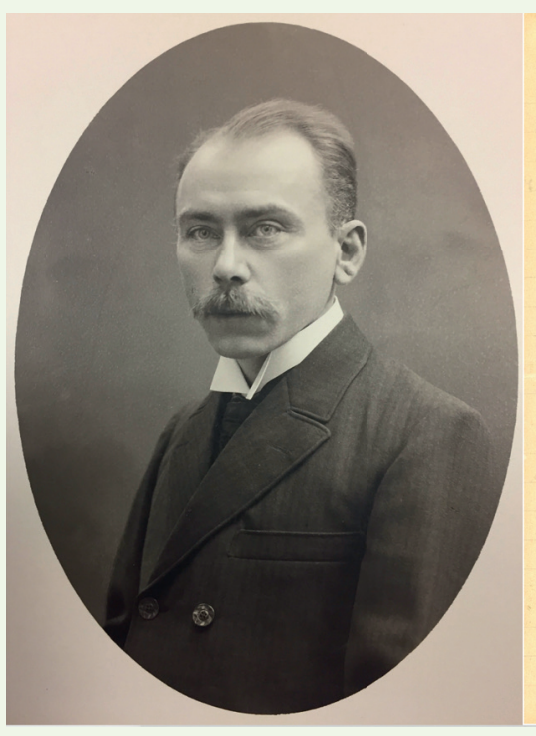

B

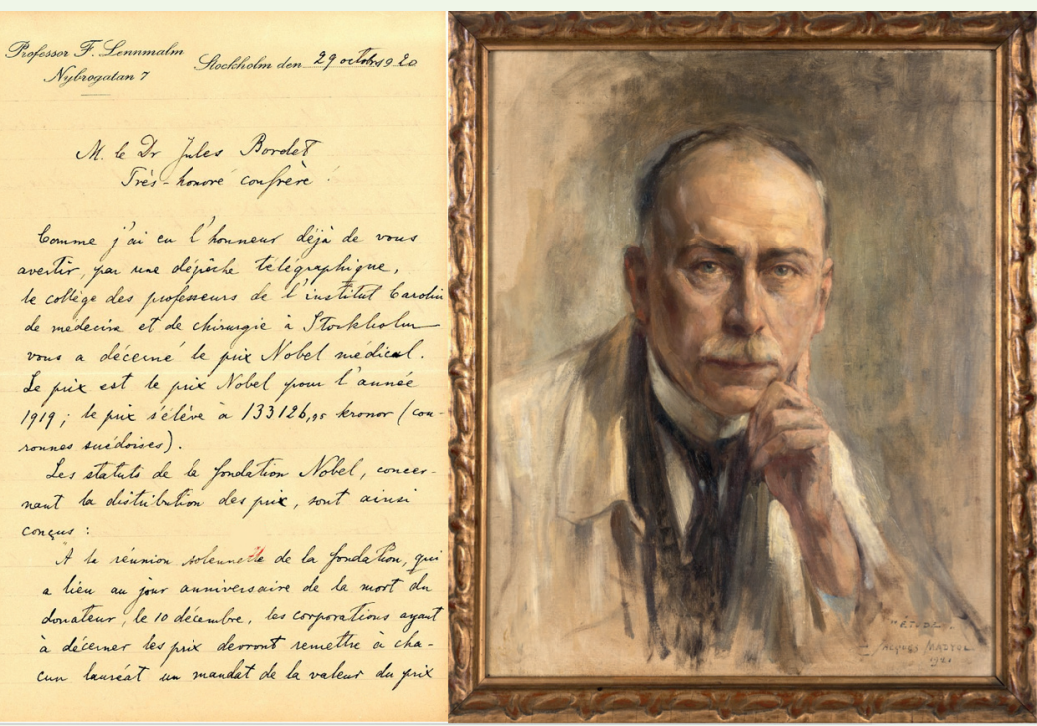

Figure 1. A. Jules Bordet vers 1901. Centre : première page de la lettre en date du 29 octobre du Prof. Lennmalm (Karolinska Institute) informant Jules Bordet de l'attribution de son prix Nobel. B. Étude pour le tableau de Jules Bordet par Jacques Madyol (1921) (๔ Documents archives et phototèque de l'Institut Pasteur).

rale, l'immunité de ses concurrents, peut-être avec l'idée de démontrer qu'elle était moins importante que l'immunité cellulaire. Mais, au sein du laboratoire du défenseur du rôle majeur joué par l'immunité cellulaire, Bordet découvrira de nouveaux mécanismes d'immunité humorale. Il démontrera l'importance du partenariat entre les anticorps et le système du complément pour lutter contre les agents infectieux. Ses découvertes vont alors également ouvrir la porte au développement d'outils de diagnostic.

Lors de son séjour parisien, deux événements importants vont se produire : son mariage avec Marthe Levoz (1876-1961), événement célébré en Belgique, et la naissance de leur première fille, Simone (1900-1983), née à Paris. Ses liens avec l'Institut parisien resteront solides après son retour en Belgique. Il est en effet revenu régulièrement pour enseigner l'immunologie et, en 1934, il sera nommé président du conseil scientifique de l'Institut Pasteur (jusqu'en 1940). En 1938, il fut fait Grand-Croix de la Légion d'honneur lors d'une cérémonie qui eut lieu dans la bibliothèque de l'Institut Pasteur, en présence du secrétaire de l'Académie des sciences française et de l'ambassadeur de Belgique.

\section{Les travaux de Jules Bordet dans le laboratoire de Metchnikoff (1894-1901)}

À l'occasion des 80 ans de Jules Bordet, le professeur Louis Pasteur Vallery-Radot (petit-fils de Louis Pasteur), évoque les années passées par Bordet à l'Institut Pasteur [8]: «Monsieur, à vingt-trois ans, vous êtes entré à l'Institut Pasteur. Vous aviez les yeux bleus où s'extériorisait magnifiquement le ciel de vos rêves, un sourire à la fois malicieux et bienveillant, qui attirait la sympathie de tous, une intonation chantante et modulée qui trahissait l'aimable pays dont vous veniez, et, sous une apparence de timidité, une assurance qui fait dire: Ce petit Jules sait ce qu'il veut, il ira loin. [...] Comme Pascal, comme Carnot, comme Lavoisier, comme Pasteur vous étiez célèbre à trente ans ». Insistant sur l'influence de ses maîtres parisiens devenus ses amis, le petit-fils de Pasteur ajouta : "Vous avez hérité du génie de Pasteur, de la clarté de Roux, de l'élégance de Duclaux». Les yeux bleus de Bordet avaient également impressionné Alexander Fleming qui, assistant à la cérémonie en tant que représentant des scientifiques étrangers, cita Paul de Kruif (1890-1971), un microbiologiste américain auteur du livre Microbes hunters : « Il était timide et semblait insignifiant, il était désordonné, il avait des yeux distraits d'un bleu d'eau, des yeux qui voyaient des choses qu'aucun autre ne cherchait à voir ». Fleming a ensuite cité Bordet, lui-même, pour illustrer sa modestie: « $\varepsilon n$ 1919, Bordet obtint le prix Nobel. Lorsqu'on le félicita de cet honneur à New York, il répondit avec sa modestie caractéristique: je dois beaucoup à l'école française de Pasteur qui m'a initié à la recherche scientifique ». Nul doute que le séjour de Bordet à l'Institut Pasteur, proche de certains des scientifiques français les plus brillants, dans le laboratoire de l'un des scientifiques les plus étonnants et les plus remarquables, Elie Metchnikoff [9], a profondément influencé la trajectoire de sa vie. 


\section{La bactériolyse}

La première publication de Jules Bordet, relatant ses investigations dans le laboratoire de Metchnikoff, a été publiée en 1895. II s'agit d'un long document de 45 pages [10], principalement destiné à rapporter les expériences menées in vitro, afin d'étudier le « phénomène de Pfeiffer », une bactériolyse observée in vivo. Pour Metchnikoff, cette observation était principalement due aux phagocytes, alors que Pfeiffer considérait qu'elle était le reflet de l'activité bactéricide des humeurs. Dans ce manuscrit, une hypothèse très étrange est émise: «Le virus capturé par les phagocytes est transporté par eux dans les organes internes où il s'accumule ». Une telle déclaration a été soutenue par des scientifiques allemands, comme Rudolph Emmerich (1856-1914), contre lesquels Metchnikoff se battait. Plus intéressant encore, dans son article, Bordet se réfère au travail de Denys qui avait découvert en 1895 le processus d'opsonisation: "Chez le lapin vacciné, les leucocytes tirent du sérum leur pouvoir d'engloutir et de détruire Streptococcus pyogenes » [11]. Si le mot opsonisation a été créé plus tard, en 1903, par Sir Edward Almroth Wright (1861-1947), Bordet qualifie de «stimuline» I'action positive des «sérums préventifs » pour favoriser le processus de phagocytose. Cependant, pour Metchnikoff, l'opsonisation ne sera jamais un concept central pour expliquer le rôle collaboratif efficace entre les phagocytes et les facteurs sériques. Par exemple, il écrit dans une préface d'un livre publié en 1915 [12]: «Le fait que la phagocytose est souvent spontanée, indépendante de l'apport des opsonines, et le fait que les recherches sur l'action opsonique ont été réalisées in vitro, hors du corps, ne permet pas d'attribuer un rôle considérable à ce facteur humoral ». Cela contraste un peu avec l'observation et les affirmations de Bordet. Utilisant plus de 25 cobayes, quelques lapins et une chèvre, ainsi que différentes souches de vibrions cholériques et le Vibrio metschnikovii, Bordet aborde la spécificité et la réactivité croisée des sérums immuns. Plus important encore, il dissocie l'activité préventive des immunsérums (les anticorps) qui fournissent la spécificité, de l'activité bactéricide (le complément), présente aussi bien dans les sérums immuns que dans les sérums normaux, activité labile à la chaleur (elle est détruite au-dessus de $55^{\circ} \mathrm{C}$ ). Il établissait ainsi l'activité complémentaire des deux composants sériques. II reconstitua l'activité bactériolytique d'un sérum protecteur « préventif » traité par la chaleur par l'addition d'un sérum normal frais. Mais il conclut également que l'activité bactéricide dérivait des leucocytes. Cette idée se perpétuera dans l'équipe de Metchnikoff et, alors qu'ils observaient très probablement les conséquences de la nétose $e^{2}$ et l'action des peptides cationiques, Metchnikoff et ses collaborateurs ont maintenu l'idée que les neutrophiles morts contribuaient à l'activité bactéricide trouvée dans les sérums [13]. Il est vrai que l'activité bactériolytique de lysats leucocytaires (dont les neutrophiles) avait été observée par de nombreux autres chercheurs [14-16].

L'année suivante, Bordet publie un article entièrement consacré à la phagocytose, la spécialité du laboratoire qui l'accueille [17]. Travail-

${ }^{2}$ Un des mécanismes d'action de lutte du polynucléaire neutrophile contre les microbes. Consiste en la production de filets (NET) constitués d'ADN et de protéines. lant in vivo chez le cobaye ou in vitro avec des exsudats péritonéaux, il compare la capacité phagocytaire des cellules face à un ensemble d'une douzaine de bactéries différentes. II montre que la rapidité, l'intensité et l'activité bactéricide varient selon la nature des bactéries. La même année, Bordet publie un nouvel article portant spécifiquement sur l'immunité humorale, consacré au mode d'action des sérums «préventifs » [18]. Dans cet article, il rend hommage à son maître: «Nous serons amenés à citer à maintes reprises les travaux de M. Metchnikoff, dont l'expérience et les précieux conseils profitent largement à ceux qui travaillent avec lui. Que notre cher et respecté maître reçoive ici l'expression de toute notre reconnaissance ». II est intéressant de noter que Bordet fait référence au principe d'agglutination, un mot créé par Gruber en 1896 [19] qui concerne un mécanisme déjà rapporté dès 1889 par Albert Charrin (1856-1907) [20], et qui pourra être utilisé pour le diagnostic. II fait également référence à l'hémagglutination (bien que le mot n'ait pas encore été utilisé), un phénomène similaire à l'agglutination des bactéries par les sérums immuns, mais concernant les hématies. Se référant à l'immunité active et passive, il conforte l'idée défendue par Metchnikoff : « Quant aux humeurs, elles fonctionnent lorsqu'elles transforment les vibrions et les altèrent profondément, comme dépositaires de principes actifs venant des leucocytes. S'il est vrai qu'elles peuvent dans certains cas décimer beaucoup de vibrions, la phagocytose intervient néanmoins toujours puissamment, et agissant en dernier ressort pour la destruction définitive du virus ». Alors qu'il considérait encore une fois que l'alexine est contenue dans les leucocytes et qu'ils la diffusent, il conclut que l'immunité passive augmente le pouvoir bactéricide phagocytaire.

\section{Hémagglutination et hémolyse}

Les années suivantes, Bordet concentre ses efforts sur le déchiffrement du mécanisme de l'agglutination des globules rouges par les anticorps, et l'hémolyse par les sérums immuns [21-23]. II injecte du sang de lapin dans la cavité péritonéale de cobayes et montre ainsi que ce qui est connu pour les propriétés des sérums immuns contre les vibrions, s'applique complètement aux globules rouges. Deux éléments sont impliqués : l'un, déjà présent dans le sérum normal, thermosensible, contribue à l'hémolyse ; l'autre facteur est présent dans le sérum immun et contribue, lui, à l'hémagglutination des érythrocytes. Bien sûr, si le processus d'(hém)agglutination reflète manifestement la multivalence des anticorps, ce concept n'était pas dans l'esprit des chercheurs à la fin du XIXe siècle. Bordet a également préparé des 
sérums immuns contre le lait. II montre leur capacité à former des agglomérats. II conclut que l'hémagglutination est un processus similaire au processus de coagulation. Immunisant des lapins contre les globules rouges de poulet, il montre la capacité des sérums immuns obtenus chez ces lapins à agglutiner et à lyser ces cellules, laissant seul leur noyau intact. In vivo, ces cellules sont phagocytées par les macrophages. Mélangeant du sang de lapin avec des sérums de cobaye désactivés par la chaleur («décomplémentés ») mais contenant des anticorps anti-globules rouges de lapin, il montre que l'alexine de lapin peut contribuer à la lyse de ses propres globules rouges sensibilisés. Des démonstrations similaires sont alors faites avec des sérums de rat, de chèvre et de chien comme source d'alexine. Il avait déjà remarqué que le sérum de cobaye était la source la plus efficace d'alexine. En revanche, l'alexine de poulet ne pouvait pas compléter l'activité des anticorps de lapin ou de cobaye anti-globules rouges de lapin. Bordet arrive ainsi à la conclusion que l'alexine impliquée dans la bactériolyse était la même que l'alexine impliquée dans l'hémolyse. II montre ensuite que les anticorps sont dirigés contre le stroma des globules rouges, car il établit que l'immunisation avec le stroma des globules rouges se traduit par une activité hémolytique similaire à celle du sang total. II montre que les anticorps anti-érythrocytes sont mortels lors de l'injection intra-veineuse chez le lapin. Mais lors de l'injection sous-cutanée, il obtient un sérum anti-hémolytique et établit qu'un tel antisérum neutralise à la fois la fraction sensibilisante et l'alexine [24]. II montre également que l'anti-alexine générée est spécifique de l'espèce. Ces travaux qui établissaient qu'en dépit d'une apparence similaire, tous les globules rouges de différentes espèces sont différents et peuvent être spécifiquement reconnus par des anticorps, ont ouvert la voie à de nouvelles investigations menées par Karl Landsteiner (1868-1943) (prix Nobel de physiologie ou médecine en 1930), qui découvrit les groupes sanguins [25]. La capacité à reconnaître les espèces animales selon leurs globules rouges a dès lors été mise à profit pour des investigations médico-légales.

\section{Un travail collaboratif avec 0 ctave Gengou}

Octave Gengou (1875-1957), médecin et bactériologiste belge, a rejoint Bordet dans le laboratoire de Metchnikoff. Épousant en juillet 1903 Berthe Levoz (1872-1941), la sœur de la femme de Bordet, il devient son beau-frère. En 1901, tous deux ont montré que les antisérums préparés contre le sang provenant de différentes espèces animales, contiennent également des anticorps capables de neutraliser le processus de coagulation [26]. Tous deux démontrent également que les sérums immuns dirigés contre diverses bactéries, inactivés par la chaleur, peuvent être bactéricides lorsqu'ils sont complétés par du sérum de cobaye frais [27]. II convient de noter que certains des sérums immuns étaient d'origine équine, confirmant que l'alexine n'a pas de spécificité d'espèce. Une démonstration similaire fut réalisée avec des sérums provenant de patients se remettant d'une fièvre typhoïde. Bordet et Gengou ont élégamment démontré la consommation de l'alexine par un mélange de bactéries et d'antisérum spécifique. Lorsqu'un tel sérum est mélangé avec un mélange décomplémenté par la chaleur des globules rouges, et de l'antisérum, aucune hémolyse n'est observée, illustrant que l'alexine est consommée par le complexe antigèneanticorps. Une telle approche fut valorisée par August von Wassermann (1866-1925) et ses collègues allemands pour le diagnostic de la syphilis [28]. Elle est connue sous le nom de test Bordet-Wassermann dans certains pays.

Toujours en 1901, Bordet remet en question le mécanisme d'interaction entre la substance sensibilisante et l'alexine [29]. Pour lui, une fois que les anticorps (la substance sensibilisante) sont liés à leur cible, ils permettent à l'alexine de s'y fixer. Pour ses concurrents allemands, Ehrlich et Marshall, une fois que les anticorps ont interagi avec leur cible, ils réagissent directement avec l'alexine [30]. Dans son article, Bordet réaffirme son attachement au mot alexine: «Il est donc opportun de renoncer à ces appelations de zwischenkörper, amboreceptor, complément, termes dont on fait le choix sous l'empire d'idées théoriques sûrement ingénieuses, capables, par les expériences qu'elles ont inspirées, de faire avancer la science, mais que l'expérience ne justifie point ».

Lorsque I'on travaillait dans le laboratoire de Metchnikoff, il était difficile de concevoir qu'un phénomène immunitaire puisse être totalement indépendant des leucocytes, en particulier des phagocytes. Gengou a abordé la question de l'origine de l'alexine. II a donc comparé l'activité du plasma et celle du sérum. Le plasma étant moins riche en alexine que le sérum, il conclut que les leucocytes étaient bien la source de l'alexine [31], aboutissant aux mêmes conclusions que beaucoup d'autres avant lui.

Dans un article écrit, une fois de retour à Bruxelles, avec Frederick Parker Gay (1874-1939), scientifique invité des États-Unis qui deviendra le huitième président de l'American association of immunologists (entre 1921 et 1922), Bordet résume son concept [32] : «Les expérimentateurs qui ont étudié l'hémolyse professent des idées très divergentes au sujet des relations qui s'établissent entre le globule sensibilisé et les substances actives, sensibilisatrices (ambocepteur) et alexine (complément). Il est bien connu d'abord que les globules fixent la sensibilisatrice (Ehrlich et Morgenroth), ensuite que les globules ainsi modifiés ont acquis le pouvoir, qu'ils ne possédaient auparavant, d'absorber l'alexine avec une énergie telle qu'ils peuvent en dépouiller complètement le liquide ambiant (Bordet)».

\section{Sérothérapie}

La peste bovine, bien qu'éradiquée en Europe à la fin du XIXe siècle, était toujours présente en Asie et au Moyen-Orient. Elle atteint l'Égypte en 1875 et l'Afrique 


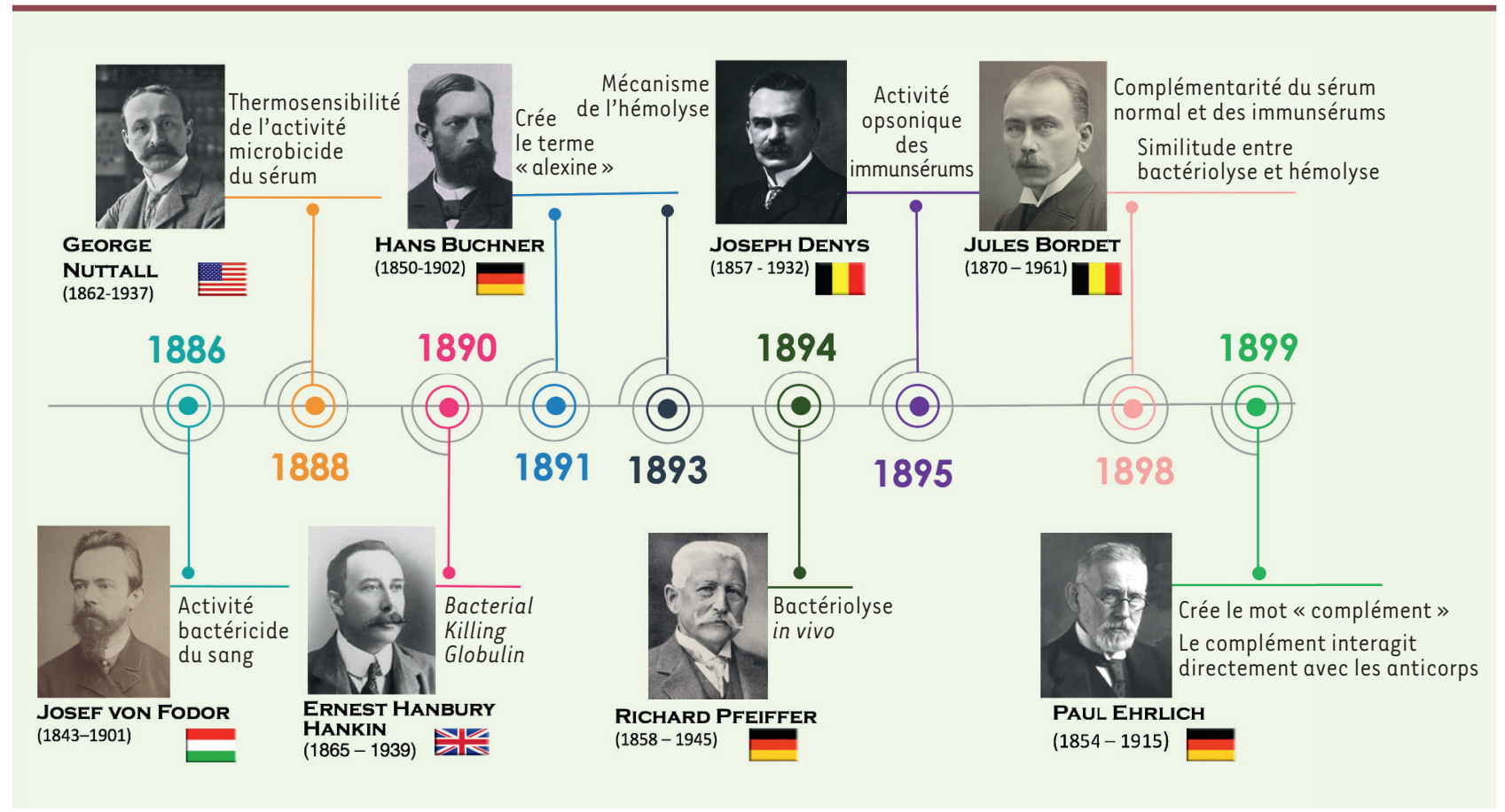

Figure 2. Les pères fondateurs du système du complément et leurs contributions majeures [42-51].

du Sud en 1896. En décembre 1896, Robert Koch rejoint Kimberley pour résoudre le problème. Jan Danysz, un bactériologiste polonais, chef de service à l'Institut Pasteur, accompagné de Jules Bordet, furent envoyés à Prétoria, capitale du Transvaal ${ }^{3}$, pour entamer une mission scientifique. Atteignant les lieux le 15 janvier 1897, ils reçurent localement l'aide d'Arnold Theiler, un jeune vétérinaire suisse qui occupait un poste officiel au sein des autorités du Transvaal [33]. Robert Koch préconisait l'injection de bile provenant d'un bœuf tué 10 à 12 jours après son infection. Mais ce traitement ne fut pas concluant. En Namibie, Paul Kohlstock (1861-1901) qui avait accompagné Koch, utilisa le traitement avec la bile. II tua 2700 des 6200 animaux traités [33]. À l'opposé, Bordet et ses deux collègues concentrèrent leurs efforts sur l'usage de la sérothérapie. Collectant du sang et du sérum d'animaux infectés survivants, ils offrirent un traitement curatif efficace. Ironiquement, l'approche fut appelée «la méthode des médecins français » [34]. Alors que leur contrat devait prendre fin le $1^{\text {er }}$ septembre 1897, le gouvernement du Transvaal leur demanda de rester pour lutter contre la peste équine. Ils revinrent en Europe début 1898, sans avoir réussi à soigner les chevaux. Dans leur rapport, Danysz et Bordet [35] décrivent le traitement mis en place lors de leur arrivée à Prétoria.

${ }^{3}$ La colonie du Transvaal était le nom d'une colonie britannique sud-africaine de 1902 à 1910.
II consiste en une injection d'un mélange de sang, de bile, de sérosité péritonéale et de contenu stomacal d'animaux morts de peste bovine, administré par voie sous-cutanée. Mais la protection obtenue variait entre $5 \%$ et $90 \%$ et ils considérèrent que cette méthode de traitement contribuait à la propagation de la maladie. Faisant référence au travail d'Eižens Zemmers (1843-1906), un vétérinaire lettonien qui fut un précurseur dans l'utilisation de la sérothérapie [36], Danysz et Bordet effectuent alors différentes expériences sur des bœufs et établissent que 1) le sang défibriné conduit à de meilleurs résultats que le sérum; 2) le volume d'inoculation doit être supérieur ou égal à $80 \mathrm{cc}$; et 3) le sang doit être prélevé deux fois après la guérison des animaux. Au total, ils mentionnent le traitement de 336 bœufs infectés, dont 309 ont été guéris, tandis que 27 ont décédé.

\section{La bactériologie}

En 1897, Bordet publia une analyse exhaustive de l'immunité contre le streptocoque [37]. II y indique l'extrême sensibilité des lapins et la sensibilité plus faible des cobayes. Bordet décrit les nombreuses propriétés de cette bactérie, comme sa capacité à empêcher la phagocytose. Une observation similaire avait déjà été faite dans le laboratoire de Metchnikoff lorsque Nicolaï Tchistovitch (1860-1926) avait étudié la phagocytose de Pasteurella multicoda par les macrophages alvéolaires. Depuis, il a été établi que la protéine $M$, la protéine $H$ et la protéine apparentée $M$, contribuaient au mécanisme d'évasion de la phagocytose par le streptocoque. II mentionne également que la bactérie a la capacité de survivre à l'intérieur du phagocyte, un mécanisme pathogène aujourd'hui bien reconnu. II signale également la 
capacité du streptocoque à interférer avec l'activité bactéricide des sérums, une fonction aujourd'hui démontrée comme étant le reflet de la capacité de certaines protéines streptococciques à limiter l'activation du complément. Enfin, il mentionne l'hémolyse observée lors de ce type d'infection, illustrant la capacité de ce pathogène à libérer de I'hémolysine. En étudiant les sérums immuns, il montre également leur faible activité bactéricide et leur faible capacité à induire une agglutination. II déclare: «Les macrophages ne se bornent pas à englober des streptocoques pour leur propre compte, ils englobent aussi des polynucléaires plus ou moins dégénérés, et qui antérieurement s'étaient emparés de microbes ». Ce que Bordet décrit, aujourd'hui connu sous le nom d'efférocytose, avait déjà été décrit dans le laboratoire de Metchnikoff par Marc Armand Ruffer (1859-1917), sept ans plus tôt.

À Paris, la fille de Bordet, âgée de 5 mois, développa une coqueluche. Il découvrit alors la présence dans ses expectorations du micro-organisme responsable de la maladie. II ne put cependant l'isoler qu'en 1906, lorsque son fils, Paul, eut également la coqueluche. Alors, avec Gengou, ils définirent un milieu de culture approprié qui leur permit d'isoler le bacille. Le milieu préparé à partir de pommes de terre, de sérum physiologique, de gélose et de sang de lapin s'est également révélé convenir à d'autres micro-organismes [38]. Bordet et Gengou ont précisé que la bactérie était particulièrement abondante au début de la maladie, tandis que sa fréquence diminuait rapidement. Ils démontrèrent que le micro-organisme isolé pouvait être mortel lorsqu'il était utilisé à des concentrations élevées chez les cobayes. Ils montrèrent que les sérums d'enfants convalescents pouvaient agglutiner les bactéries, et, en utilisant leur approche de consommation d'alexine, ils confirmèrent la présence d'anticorps spécifiques dans le sérum des enfants. L'antériorité du travail de nos deux médecins belges fut contestée par Reyher, de l'hôpital de la Charité à Berlin [39]. Bordet et Gengou demandèrent à Reyher de leur envoyer sa bactérie isolée pour permettre une comparaison fine, mais Reyher refusa. Dans leur réponse, Bordet et Gengou démontrèrent le caractère inapproprié de son affirmation, arguant des différences en termes de culture, de cellules cibles, de l'absence de démonstration avec du sérum immun, du manque d'expériences effectuées sur l'animal et se moquèrent de son utilisation de «très probablement » pour qualifier les bactéries identifiées comme celles de la coqueluche [40]. Ils terminèrent leur réponse par un commentaire cinglant: «Nous estimons en terminant cette longue discussion que les colonnes de ces Annales, notre temps et sûrement aussi celui de Mr Reyner, auraient pu être plus utilement employés ». Le nom Bordetella pertussis donné au pathogène de la coqueluche illustre le fait que la communauté scientifique reconnut Jules Bordet comme étant celui qui avait fait la première découverte et qui avait identifié correctement le germe.

\section{Le retour en Belgique}

Le 15 mars 1900, le conseil provincial du Brabant vota la création d'un institut antirabique et bactériologique. Bordet fut invité à en prendre la direction. Le 10 avril 1961, lors de ses funérailles nationales, Jacques Tréfouël, représentant l'Institut Pasteur, résumait les senti- ments des Pasteuriens parisiens lorsque Bordet décida de retourner en Belgique: "C'est dans notre institut, à Paris, que ses très jeunes années se sont écoulées, près de Metchnikoff, de Besredka, de son grand ami, M. Calmette, et de son maître vénéré M. Roux. Tous, ils avaient l'illusion que cette collaboration, parce qu'elle était trop fraternelle, fructueuse et vivifiante, pouvait être éternelle, et ce ne fut pas sans déchirement qu'ils virent repartir celui dont la place, restée vide, ne fut jamais tout à fait reprise. Il fallait, à ce réel chagrin, des dédommagements et le professeur Bordet a su nous combler. L'institut du Brabant dont il fut le fondateur et l'âme pendant de si longues années, il le considéra comme un second institut Pasteur». En Belgique, Bordet poursuivit une longue carrière, s'intéressant en particulier aux bactériophages, à l'anaphylaxie et aux propriétés bactériolytique du lait [41]. $\diamond$

\section{SUMMARY}

Jules Bordet, a man of conviction. Centenary of his Nobel Prize

Jules Bordet came to the Institut Pasteur soon after his MD graduation at the Université libre de Bruxelles, thanks to a grant from the Belgian government. He joined there the laboratory of Elie Metchnikoff, the father of phagocytes and cellular immunity. Amazingly, he will decipher there some of the key mechanisms of humoral immunity initially discovered by the German school against which his mentor was fighting. He described the mechanisms that govern bacteriolysis and hemolysis, following the action of immune sera. Even if he favored the term alexin coined by Hans Buchner, he is indeed one of the founding fathers of the complement system (term coined by Paul Ehrlich). It is for these works that he was awarded in October 1920 the 1919 Nobel Prize. Back in Belgium, he became the director of Institut Pasteur du Brabant and made another landmark discovery, namely the identification of the bacillus of whooping cough, now named Bordetella pertussis. $\diamond$

\section{LIENS D'INTÉR $\hat{T}$}

Les auteurs déclarent n'avoir aucun lien d'intérêt concernant les données publiées dans cet article.

\section{RéFÉRENCES}

1. Schmalstieg FC Jr, Goldman AS. Jules Bordet (1870-1961) : a bridge between early and modern immunology J Med Biograph 2009; 17 : 217-24.

2. Massart J, Bordet C. Recherche sur l'irritabilité des leucocytes. J Med Chirurg Pharmacol $1890 ; 90: 169-82$.

3. Massart J, Bordet C. Le chemiotaxis des leucocytes et l'infection microbienne. Ann Inst Pasteur 1891 ; 5 : 417-44.

4. Cavaillon JM, Legout S. Duclaux, Roux, Grancher, Chamberland, and Metchnikoff : the five musketeers of Louis Pasteur. Microbes Infection 2019; 21: 192-201 


\section{RÉFÉRENCES}

5. Bordet J. Adaptation des virus aux organismes vaccinés. Ann Inst Pasteur $1892 ; 6: 328-34$.

6. Metchnikoff $\varepsilon$. L'état actuel de la question de l'immunité (rapport au congrès international de Budapest. Ann Inst Pasteur 1894 ; 8 : 706-21.

7. Cavaillon JM.The historical milestones in the understanding of leukocyte biology initiated by Elie Metchnikof. J Leuk Biol 2011 ; 90 : 413-24.

8. Bordet J. Volume jubilaire de Jules Bordet (25 novembre 1950). Ann Inst Pasteur $1950 ; 79: 479-520$.

9. Cavaillon JM, Legout S. Centenary of the death of Elie Metchnikoff : a visionary and an outstanding team leader. Microbes Infection 2016; 18: 577-94.

10. Bordet J. Les leucocytes et les propriétés actives du sérum chez les vaccinés. Ann Inst Pasteur 1895 ; 9 : 462-506.

11. Denys J, LeclefJ. Sur le mécanisme de l'immunité. La Cellule 1895; 11 : 175-221.

12. Petit R. Les phagocytes en chirurgie. Paris : Masson ; 1915.

13. Levaditi C. Sur l'état de la cytase dans le plasma des animaux normaux et des organismes vaccinés contre le vibrion cholérique. Ann Inst Pasteur $1901 ; 15: 894-927$.

14. Denys J, Havet J. Sur la part des leucocytes dans le pouvoir bactéricide du sang de chien. La Cellule $1894 ; 10: 7-35$

15. Schattenfroh A. Ueber die backerienfeindlichen eigenschaften der leukocyten. Arch Hyg $1897 ; 31$ : 1-81.

16. Hahn M. Ueber die beziehungen der leucocyten zur bactericiden wirkung des blutes. Arch Hyg $1895 ; 25$ : 105-44.

17. Bordet J. Recherche sur la phagocytose. Ann Inst Pasteur 1986 ; 10 : 104-18.

18. Bordet J. Sur le mode d'action des sérums préventifs. Ann Inst Pasteur 1896 ; $10: 193-219$.

19. Gruber, M., Über active und passive immunität gegen cholera und typhus, sowie über die bacteriologische diagnose der cholera und des typhus. Wien Klin Wochenschr 1896 ; ix : 183-6 et 204-9.

20. Charrin $A$. Roger, Note sur le développement des microbes pathogènes dans le sérums des animaux vaccinés. CR Soc Biol $1889 ; 41: 667-9$.

21. Bordet J. Sur l'agglutination et la dissolution des globules rouges par le sérum d'animaux injectés de sang défibriné. Ann Inst Pasteur $1898 ; 12$ : 688-95.

22. Bordet J. Le mécanisme de l'agglutination. Ann Inst Pasteur 1899 ; 13 22550

23. Bordet J Agglutination et dissolution des globules rouges par le sérum. Ann Inst Pasteur $1899 ; 13: 273-87$.

24. Bordet J. Les sérums hémolytiques, leurs antitoxines et les théories des sérums cytolytiques. Ann Inst Pasteur $1900 ; 14: 257-73$

25. Landsteiner $K$. Uber agglutinationerscheinungen normalen menschlichen blutes. Wien Klin Wochschrft 1901; 14 : 1132-4.

26. Bordet J, Gengou, 0 . Recherche sur la coagulation du sang et les sérums anti-coagulants. Ann Inst Pasteur $1901 ; 15$ : 129-44.
27. Bordet J, Gengou, 0 . Sur l'existence de substances sensibilisatrices dans la plupart des sérums antimicrobiens. Ann Inst Pasteur 1901; 15 : 289-302.

28. Von Wassermann A, Neisser A, Bruck C. Eine serodiagnostische reaktion bei syphilis. Deut Med Wochschrft $1906 ; 32: 745-6$.

29. Bordet J. Sur le mode d'action des sérums cytolytiques et sur l'unité de l'alexine dans un même sérum. Ann Inst Pasteur $1901 ; 15: 303-18$.

30. Ehrlich P, Marshall HT Ueber die complementophilen gruppen der amboceptoren. Berliner klinische Wochenschrift $1902 ; 39: 585-7$.

31. Gengou 0. Contribution à l'étude des l'origine de l'alexine des sérums normaux. II. L'alexine des sérums normaux est-elle un produit de secrétion des globules blancs? Ann Inst Pasteur 1901 ; $15: 232-48$.

32. Bordet J, Gay FP. Sur les relations des sensibilisatrices avec l'alexine. Ann Inst Pasteur 1906 ; 20 : 467-498.

33. Mammerickx M. La peste bovine, Jules Bordet et le centre sérumigène de Cureghem. Ann Med Vet $2003 ; 147: 197-205$

34. Mack R. The great African cattle plague epidemic of the 1890's. Trop Anim HIth Prod. $1970 ; 2$ : $210-9$

35. Danysz J., Bordet J. Rapport sur les recherches concernant la peste bovine. Bull Agric 1898 ; 14 : 77-88.

36. Eižens N. Zemmers EN. Rinderpest-infektion und immunisierung und schutzimpfung gegen rinderpest. Berliner Tierärztliche Wochenschrift 1893; $23: 590-1$.

37. Bordet J. Contribution à l'étude du sérum antistreptococcique. Ann Inst Pasteur 1897 ; 11 : 177-263.

38. Bordet J, Gengou 0. Le microbe de la coqueluche. Ann Inst Pasteur 1906; $20: 731-41$

39. Reyher. Le microbe de la coqueluche. Ann Inst Pasteur 1907 ; 21 : 727-32.

40. Bordet J, Gengou 0. Le microbe de la coqueluche. Ann Inst Pasteur $1907 ; 21: 733-8$.

41. Cavaillon JM, Sansonetti P, Goldman M. 100th Anniversary of Jules Bordet's Nobel Prize : tribute to a founding father of immunology. Front Immunol $2019 ; 10: 2114$

42. Fodor J. Bakterien im blute lebender thiere. Arch Hyg $1886 ; 4$ : 129-48

43. Fodor J. Die fahigkeit des blutes bakterien zu vernichten. Dtsch Med Woch 1887 ; 8 : 745-747

44. Nuttall G. Experimente uber die bacterienfeindlichen einfluesse des thierischen korpers. $Z$ Hyg Infektkr $1888 ; 4: 353-94$

45. Buchner $\mathrm{H}$. Ueber die bakterientödtende wirkung des zellenfreien blutserums. Zbl Bakt Parasitol $1889 ; 5: 817-23$ et $6: 1-11$

46. Buchner Kurze. Uebersicht über die entwicklung der bakterienforschung seitnaegeli's eingreifen in dieselbe. Vortrag, gehalten im aerztlichen vereine in München am 3. Juni 1891. Münchene Medizinische Wochenschrift $1891 ; n^{\circ} 25$ (S435) et 26 (S454)

47. Hankin $\varepsilon H$. Ueber die nomenclatur der schützenden eiweisskörper. Zbl Bakt Parasitol $1891 ; 10$ : 377-79.

48. Hankin. A bacterial killing globulin. Proc $R$ Soc London $1890 ; 48$ : 93-101

49. Pfeiffer R. Weitere untersuchungen über das wesen der choleraimmunität und über specifische processe. Z Hyg Infektkrabkh 1894 ; 18 : 1-16

50. Denys J, Havet J. Sur la part des leucocytes dans le pouvoir bactéricide du sang de chien. La Cellule $1894 ; 10: 7-35$.

51. Ehrlich P, Morgenroth J. Ueber haemolysine : zweite mittheilung. Berliner klinische Wochenschrift 1899 ; 36 : 481-6.

\section{TIRÉS À PART}

J.M. Cavaillon

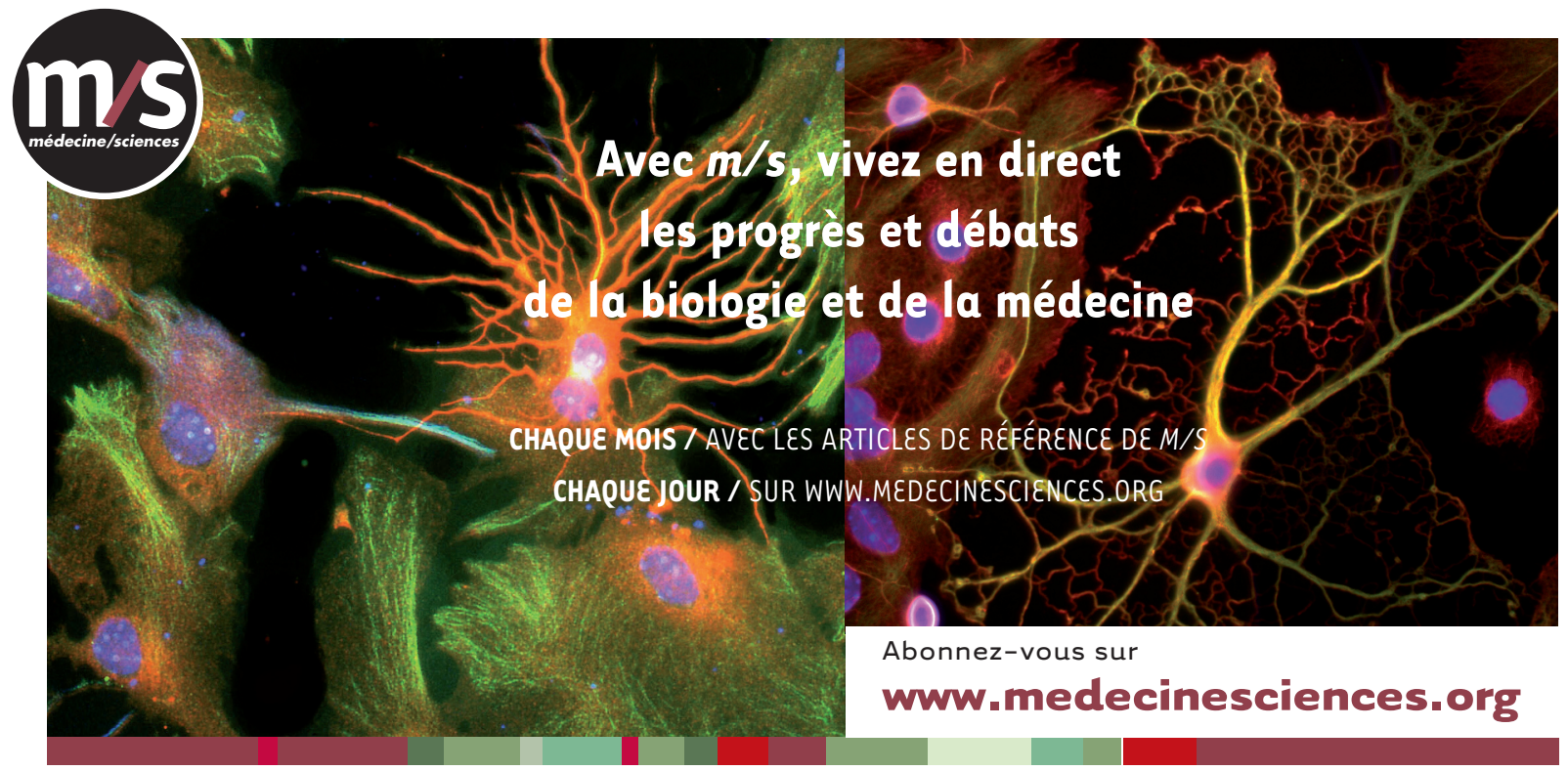

